\title{
Relativistic dissipative hydrodynamics from kinetic theory with relaxation time approximation
}

\author{
Amaresh Jaiswal \\ Tata Institute of Fundamental Research, Homi Bhabha Road, Mumbai 400005, India
}

(Dated: October 30, 2018)

\begin{abstract}
Starting from Boltzmann equation with relaxation time approximation for the collision term and using Chapman-Enskog like expansion for distribution function close to equilibrium, we derive hydrodynamic evolution equations for the dissipative quantities directly from their definition. Although the form of the equations is identical to those obtained in traditional Israel-Stewart approaches employing Grad's 14-moment approximation and second moment of Boltzmann equation, the coefficients obtained are different. In the case of one-dimensional scaling expansion, we demonstrate that our results are in better agreement with numerical solution of Boltzmann equation as compared to Israel-Stewart results. We also show that including approximate higher-order corrections in viscous evolution significantly improves this agreement, thus justifying the relaxation time approximation for the collision term.
\end{abstract}

PACS numbers: 25.75.Ld, 24.10.Nz, 47.75+f, 47.10.ad

Relativistic fluid dynamics has been applied quite successfully to study and understand a wide range of collective phenomena observed in cosmology, astrophysics and the physics of high-energy, heavy-ion collisions. The earliest theoretical formulation of relativistic dissipative hydrodynamics also known as first-order theories (order of gradients), are due to Eckart [1] and Landau-Lifshitz [2]. However these theories, collectively called relativistic Navier-Stokes (NS) theory, involve parabolic differential equations and suffer from acausality and numerical instability. The Chapman-Enskog (CE) expansion has been the most common method to obtain first-order hydrodynamics from Boltzmann Equation (BE) [3]. The derivation of second-order fluid-dynamics by Israel and Stewart (IS) from kinetic theory uses extended Grad's method [4]. The approach by Israel and Stewart may not guarantee stability but solves the acausality problem [5] at the cost of introducing two additional approximations: (a) 14-moment approximation for the distribution function and, (b) use of second moment of BE to obtain evolution equations for dissipative quantities.

Grad's method, originally proposed for non-relativistic systems, was modified by Israel and Stewart so that it could be applicable to the relativistic case. In this extension, known as 14-moment approximation, the distribution function is Taylor expanded in powers of fourmomenta around its local equilibrium value. Truncating the Taylor expansion at second-order in momenta results in 14 unknowns that have to be determined to describe the distribution function. This expansion implicitly assumes a converging series in powers of momenta. In addition, it is assumed that the order of expansion in 14-moment approximation (expanded as a series in momenta) coincides with that of gradient expansion of hydrodynamics. This is evident because Grad's approximation truncated at second-order in momenta is not consistent with second-order hydrodynamics.

Another assumption inherent in IS derivation is the choice of second moment of the BE to extract the equa- tion of motion for the dissipative quantities. This choice is arbitrary in the sense that once the distribution function is specified, any moment of the BE will lead to a closed set of equations for the dissipative currents but with different transport coefficients. In fact, it has been pointed out in Ref. [6] that instead of this ambiguous choice of the second-moment of BE by IS, the dissipative quantities can be obtained directly from their definition. Consistent and accurate formulation of relativistic dissipative hydrodynamics is still unresolved and is currently an active research area 6 -10].

In this Rapid Communication, we present an alternative derivation of hydrodynamic equations for dissipative quantities which do not make use of both these assumptions. We revisit the CE expansion of the distribution function using BE in Relaxation Time Approximation (RTA). Using this expansion, we derive the first and second-order equations of motion for the dissipative quantities from their definition. In one-dimensional boost-invariant Bjorken scenario, we demonstrate that our second-order results are in better agreement with transport results as compared to those obtained by using IS equations. We also illustrate that heuristic incorporation of higher-order corrections in viscous evolution equation significantly improves this agreement.

Fluid dynamics is best described as a long-wavelength, low-frequency limit of an underlying microscopic theory. Further, BE governs the temporal evolution of single particle phase-space distribution function $f \equiv f(x, p)$ which provides a reliably accurate description of the microscopic dynamics in the dilute limit. With this motivation, our starting point for the derivation of hydrodynamic equations is relativistic BE with RTA for the collision term [11]

$$
p^{\mu} \partial_{\mu} f=-\frac{u \cdot p}{\tau_{R}}\left(f-f_{0}\right),
$$

where, $p^{\mu}$ is the particle four-momentum, $u_{\mu}$ is the fluid four-velocity and $\tau_{R}$ is the relaxation time. We define the 
scalar product $u \cdot p \equiv u_{\mu} p^{\mu}$. With $f \rightarrow \bar{f}$ and $f_{0} \rightarrow \bar{f}_{0}$, Eq. (11) describes the evolution of distribution function for antiparticles. The equilibrium distribution functions for Fermi, Bose, and Boltzmann particles $(r=1,-1,0)$ are

$$
f_{0}=\frac{1}{\exp (\beta u \cdot p-\alpha)+r},
$$

and $\alpha \rightarrow-\alpha$ for antiparticles $\bar{f}_{0}$. Here, $\beta=1 / T$ is the inverse temperature and $\alpha=\mu / T$ is the ratio of chemical potential to temperature.

In the $\mathrm{CE}$ expansion, the particle distribution function is expanded about its equilibrium value in powers of space-time gradients.

$$
f=f_{0}+\delta f, \quad \delta f=\delta f^{(1)}+\delta f^{(2)}+\cdots,
$$

where $\delta f^{(1)}$ is first-order in gradients, $\delta f^{(2)}$ is secondorder and so on. The Boltzmann equation, (1), in the form $f=f_{0}-\left(\tau_{R} / u \cdot p\right) p^{\mu} \partial_{\mu} f$, can be solved iteratively as [12]

$$
f_{1}=f_{0}-\frac{\tau_{R}}{u \cdot p} p^{\mu} \partial_{\mu} f_{0}, \quad f_{2}=f_{0}-\frac{\tau_{R}}{u \cdot p} p^{\mu} \partial_{\mu} f_{1}, \quad \cdots
$$

where $f_{1}=f_{0}+\delta f^{(1)}$ and $f_{2}=f_{0}+\delta f^{(1)}+\delta f^{(2)}$. To first and second-order in gradients, we obtain

$$
\begin{aligned}
\delta f^{(1)} & =-\frac{\tau_{R}}{u \cdot p} p^{\mu} \partial_{\mu} f_{0} \\
\delta f^{(2)} & =\frac{\tau_{R}}{u \cdot p} p^{\mu} p^{\nu} \partial_{\mu}\left(\frac{\tau_{R}}{u \cdot p} \partial_{\nu} f_{0}\right) .
\end{aligned}
$$

The above treatment to obtain $\delta f$ is valid for $\delta \bar{f}$ (antiparticles) as well.

For the sake of comparison, we also write down the Grad's 14-moment expansion [13] in orders of momenta as suggested by IS [4] in orthogonal basis [10],

$$
\delta f=f_{0} \tilde{f}_{0}\left(\lambda_{\Pi} \Pi+\lambda_{n} n_{\alpha} p^{\alpha}+\lambda_{\pi} \pi_{\alpha \beta} p^{\alpha} p^{\beta}\right)+\mathcal{O}\left(p^{3}\right),
$$

where, $\tilde{f}_{0}=1-r f_{0}$ and $\lambda_{\Pi}, \lambda_{n}, \lambda_{\pi}$ are determined from the definition of the dissipative quantities, Eqs. (10)(12). Since hydrodynamics involves expansion in orders of gradients, hence for consistency, CE should be preferred over 14-moment approximation in derivation of hydrodynamic equations.

The conserved energy-momentum tensor and particle current can be expressed in terms of distribution function as 14.

$$
\begin{aligned}
T^{\mu \nu} & =\int d p p^{\mu} p^{\nu}(f+\bar{f})=\epsilon u^{\mu} u^{\nu}-(P+\Pi) \Delta^{\mu \nu}+\pi^{\mu \nu}, \\
N^{\mu} & =\int d p p^{\mu}(f-\bar{f})=n u^{\mu}+n^{\mu}
\end{aligned}
$$

where $d p=g d \mathbf{p} /\left[(2 \pi)^{3} \sqrt{\mathbf{p}^{2}+m^{2}}\right], g$ and $m$ being the degeneracy factor and particle mass. In the tensor decompositions, $\epsilon, P, n$ are respectively energy density, pressure, net number density, and $\Delta^{\mu \nu}=g^{\mu \nu}-u^{\mu} u^{\nu}$ is the projection operator on the three-space orthogonal to the hydrodynamic four-velocity $u^{\mu}$ defined in the Landau frame: $T^{\mu \nu} u_{\nu}=\epsilon u^{\mu}$. The metric tensor is $g^{\mu \nu} \equiv \operatorname{diag}(+,-,-,-)$. The bulk viscous pressure (П), shear stress tensor $\left(\pi^{\mu \nu}\right)$ and particle diffusion current $\left(n^{\mu}\right)$ are the dissipative quantities.

Energy-momentum conservation, $\partial_{\mu} T^{\mu \nu}=0$ and current conservation, $\partial_{\mu} N^{\mu}=0$, yields the fundamental evolution equations for $n, \epsilon$ and $u^{\mu}$

$$
\begin{aligned}
\dot{\epsilon}+(\epsilon+P+\Pi) \theta-\pi^{\mu \nu} \nabla_{(\mu} u_{\nu)} & =0, \\
(\epsilon+P+\Pi) \dot{u}^{\alpha}-\nabla^{\alpha}(P+\Pi)+\Delta_{\nu}^{\alpha} \partial_{\mu} \pi^{\mu \nu} & =0, \\
\dot{n}+n \theta+\partial_{\mu} n^{\mu} & =0 .
\end{aligned}
$$

We use the standard notation $\dot{A}=u^{\mu} \partial_{\mu} A$ for comoving derivative, $\nabla^{\alpha}=\Delta^{\mu \alpha} \partial_{\mu}$ for space-like derivative, $\theta=\partial_{\mu} u^{\mu}$ for expansion scalar and $A^{(\alpha} B^{\beta)}=$ $\left(A^{\alpha} B^{\beta}+A^{\beta} B^{\alpha}\right) / 2$ for symmetrization.

Even if the equation of state relating $\epsilon$ and $P$ is provided, the system of Eqs. (9) is not closed unless the dissipative quantities $\Pi, n^{\mu}$ and $\pi^{\mu \nu}$ are specified. To obtain the expressions for these dissipative quantities, we write them using Eq. (8) in terms of away from equilibrium part of the distribution functions $(\delta f, \delta \bar{f})$ as

$$
\begin{aligned}
\Pi & =-\frac{\Delta_{\alpha \beta}}{3} \int d p p^{\alpha} p^{\beta}(\delta f+\delta \bar{f}), \\
n^{\mu} & =\Delta_{\alpha}^{\mu} \int d p p^{\alpha}(\delta f-\delta \bar{f}), \\
\pi^{\mu \nu} & =\Delta_{\alpha \beta}^{\mu \nu} \int d p p^{\alpha} p^{\beta}(\delta f+\delta \bar{f}),
\end{aligned}
$$

where $\Delta_{\alpha \beta}^{\mu \nu}=\left[\Delta_{\alpha}^{\mu} \Delta_{\beta}^{\nu}+\Delta_{\beta}^{\mu} \Delta_{\alpha}^{\nu}-(2 / 3) \Delta^{\mu \nu} \Delta_{\alpha \beta}\right] / 2$.

The first-order dissipative equations can be obtained from Eqs. (10)-(12) using $\delta f=\delta f^{(1)}$ from Eq. (5)

$$
\begin{aligned}
\Pi & =-\frac{\Delta_{\alpha \beta}}{3} \int d p p^{\alpha} p^{\beta}\left[-\frac{\tau_{R}}{u \cdot p} p^{\gamma} \partial_{\gamma}\left(f_{0}+\bar{f}_{0}\right)\right], \\
n^{\mu} & =\Delta_{\alpha}^{\mu} \int d p p^{\alpha}\left[-\frac{\tau_{R}}{u \cdot p} p^{\gamma} \partial_{\gamma}\left(f_{0}-\bar{f}_{0}\right)\right], \\
\pi^{\mu \nu} & =\Delta_{\alpha \beta}^{\mu \nu} \int d p p^{\alpha} p^{\beta}\left[-\frac{\tau_{R}}{u \cdot p} p^{\gamma} \partial_{\gamma}\left(f_{0}+\bar{f}_{0}\right)\right] .
\end{aligned}
$$

Assuming the relaxation time $\tau_{R}$ to be independent of four-momenta, the integrals in Eqs. (13)-(15) reduce to

$$
\Pi=-\tau_{R} \beta_{\Pi} \theta, \quad n^{\mu}=\tau_{R} \beta_{n} \nabla^{\mu} \alpha, \quad \pi^{\mu \nu}=2 \tau_{R} \beta_{\pi} \sigma^{\mu \nu},
$$

where $\sigma^{\mu \nu}=\Delta_{\alpha \beta}^{\mu \nu} \nabla^{\alpha} u^{\beta}$. The coefficients $\beta_{\Pi}, \beta_{n}$ and $\beta_{\pi}$ are found to be

$$
\begin{aligned}
\beta_{\Pi}= & \frac{1}{3}\left(1-3 c_{s}^{2}\right)(\epsilon+P)-\frac{2}{9}(\epsilon-3 P) \\
& -\frac{m^{4}}{9}\left\langle(u \cdot p)^{-2}\right\rangle_{0^{+}}, \\
\beta_{n}= & -\frac{n^{2}}{\beta(\epsilon+P)}+\frac{2\langle 1\rangle_{0^{-}}}{3 \beta}+\frac{m^{2}}{3 \beta}\left\langle(u \cdot p)^{-2}\right\rangle_{0^{-}}, \\
\beta_{\pi}= & \frac{4 P}{5}+\frac{\epsilon-3 P}{15}-\frac{m^{4}}{15}\left\langle(u \cdot p)^{-2}\right\rangle_{0^{+}},
\end{aligned}
$$


where $\langle\cdots\rangle_{0^{ \pm}}=\int d p(\cdots)\left(f_{0} \pm \bar{f}_{0}\right)$, and $c_{s}^{2}=(d P / d \epsilon)_{s / n}$ is the adiabatic speed of sound squared ( $s$ being the entropy density). It is interesting to note that these coefficients are in perfect agreement with those obtained in the Ref. [6] in which the evolution equations are derived directly from their definition. This is due to the fact that in Ref. [6], the coefficients $\beta_{\Pi}, \beta_{n}$ and $\beta_{\pi}$, are associated with first-order terms and do not involve 14-moment approximation. In the massless limit, $\beta_{\pi}=4 P / 5$ is also in agreement with that obtained in Ref. [12] employing CE expansion in $\mathrm{BE}$ with medium-dependent masses.

In the process to obtain second-order equations, we discover that $\mathrm{CE}$ expansion for the distribution function does not support derivation of hydrodynamic evolution equations from arbitrary moment choice of BE. Using the definition of dissipative quantities to obtain their evolution equations comes naturally when employing $\mathrm{CE}$ expansion as demonstrated while deriving firstorder equations, Eq. (16). Second-order evolution equations can also be obtained similarly by substituting $\delta f=$ $\delta f^{(1)}+\delta f^{(2)}$ from Eqs. (5) and (6) in Eqs. (10)-(12).

$$
\begin{gathered}
\frac{\Pi}{\tau_{R}}=\frac{\Delta_{\alpha \beta}}{3} \int d p p^{\alpha} p^{\beta}\left[\frac{p^{\gamma}}{u \cdot p} \partial_{\gamma} f_{0}-\frac{p^{\gamma} p^{\rho}}{u \cdot p} \partial_{\gamma}\left(\frac{\tau_{R}}{u \cdot p} \partial_{\rho} f_{0}\right)\right. \\
\left.+f_{0} \rightarrow \bar{f}_{0}\right], \\
\frac{n^{\mu}}{\tau_{R}}=-\Delta_{\alpha}^{\mu} \int d p p^{\alpha}\left[\frac{p^{\gamma}}{u \cdot p} \partial_{\gamma} f_{0}-\frac{p^{\gamma} p^{\rho}}{u \cdot p} \partial_{\gamma}\left(\frac{\tau_{R}}{u \cdot p} \partial_{\rho} f_{0}\right)\right. \\
\left.-f_{0} \rightarrow \bar{f}_{0}\right], \\
\frac{\pi^{\mu \nu}}{\tau_{R}}=-\Delta_{\alpha \beta}^{\mu \nu} \int d p p^{\alpha} p^{\beta}\left[\frac{p^{\gamma}}{u \cdot p} \partial_{\gamma} f_{0}-\frac{p^{\gamma} p^{\rho}}{u \cdot p} \partial_{\gamma}\left(\frac{\tau_{R}}{u \cdot p} \partial_{\rho} f_{0}\right)\right. \\
\left.+f_{0} \rightarrow \bar{f}_{0}\right] .
\end{gathered}
$$

The derivatives of equilibrium distribution function $\left(\partial_{\mu} f_{0}, \partial_{\mu} \partial_{\nu} f_{0}\right)$ appearing in above equations can be obtained by successively differentiating Eq. (2). The momentum integrations can be decomposed into hydrodynamic tensor degrees of freedom via the definitions:

$$
\begin{aligned}
I_{(m) \pm}^{\mu_{1} \cdots \mu_{n}} \equiv & \int \frac{d p}{(u \cdot p)^{m}} p^{\mu_{1}} \cdots p^{\mu_{n}}\left(f_{0} \pm \bar{f}_{0}\right)=I_{n 0}^{(m) \pm} u^{\mu_{1}} \cdots u^{\mu_{n}} \\
& +I_{n 1}^{(m) \pm}\left(\Delta^{\mu_{1} \mu_{2}} u^{\mu_{3}} \cdots u^{\mu_{n}}+\text { perms }\right)+\cdots,
\end{aligned}
$$

where 'perms' denotes all non-trivial permutations of the Lorentz indices. We similarly define $J_{(m) \pm}^{\mu_{1} \mu_{2} \cdots \mu_{n}}$ where the momentum integrals are weighted with $f_{0} \tilde{f}_{0} \pm\left(f_{0} \rightarrow \bar{f}_{0}\right)$, and are tensor decomposed with coefficients $J_{n q}^{(m) \pm}$.

After performing the integration, the relaxation time appearing on the right hand side of Eqs. (20)-(22) are absorbed using the first-order equations for the dissipative quantities, Eq. (16). Using the identity $\nabla^{\mu} \beta=$ $-\beta \dot{u}^{\mu}+[n /(\epsilon+P)] \nabla^{\mu} \alpha+\mathcal{O}\left(\delta^{2}\right)$, the terms containing derivatives of the relaxation time cancel each other upto second-order in gradients and hence the right hand side of Eqs. (20)-(22) can be made independent of $\tau_{R}$ [15].
The second-order evolution equations of the dissipative quantities are finally obtained as

$$
\begin{aligned}
& \frac{\Pi}{\tau_{R}}=-\dot{\Pi}-\beta_{\Pi} \theta-\delta_{\Pi \Pi} \Pi \theta+\lambda_{\Pi \pi} \pi^{\mu \nu} \sigma_{\mu \nu} \\
& -\tau_{\Pi n} n \cdot \dot{u}-\lambda_{\Pi n} n \cdot \nabla \alpha-\ell_{\Pi n} \partial \cdot n, \\
& \frac{n^{\mu}}{\tau_{R}}=-\dot{n}^{\langle\mu\rangle}+\beta_{n} \nabla^{\mu} \alpha-n_{\nu} \omega^{\nu \mu}-\lambda_{n n} n^{\nu} \sigma_{\nu}^{\mu}-\delta_{n n} n^{\mu} \theta \\
& +\lambda_{n \Pi} \Pi \nabla^{\mu} \alpha-\lambda_{n \pi} \pi^{\mu \nu} \nabla_{\nu} \alpha-\tau_{n \pi} \pi_{\nu}^{\mu} \dot{u}^{\nu} \\
& +\tau_{n \Pi} \Pi \dot{u}^{\mu}+\ell_{n \pi} \Delta^{\mu \nu} \partial_{\gamma} \pi_{\nu}^{\gamma}-\ell_{n \Pi} \nabla^{\mu} \Pi, \\
& \frac{\pi^{\mu \nu}}{\tau_{R}}=-\dot{\pi}^{\langle\mu \nu\rangle}+2 \beta_{\pi} \sigma^{\mu \nu}+2 \pi_{\gamma}^{\langle\mu} \omega^{\nu\rangle \gamma}-\tau_{\pi \pi} \pi_{\gamma}^{\langle\mu} \sigma^{\nu\rangle \gamma} \\
& -\delta_{\pi \pi} \pi^{\mu \nu} \theta+\lambda_{\pi \Pi} \Pi \sigma^{\mu \nu}-\tau_{\pi n} n^{\langle\mu} \dot{u}^{\nu\rangle} \\
& +\lambda_{\pi n} n^{\langle\mu} \nabla^{\nu\rangle} \alpha+\ell_{\pi n} \nabla^{\langle\mu} n^{\nu\rangle},
\end{aligned}
$$

where the vorticity tensor is defined as $\omega^{\mu \nu}=\left(\nabla^{\mu} u^{\nu}-\right.$ $\left.\nabla^{\nu} u^{\mu}\right) / 2$. All the coefficients in the above equations have been obtained in terms of $\beta$ and the integral coefficients $I_{n q}^{(m) \pm}$ and $J_{n q}^{(m) \pm}$ [15]. It is clear that in Eqs. (24)-(26), the Boltzmann relaxation time $\tau_{R}$ can be replaced by those of the individual dissipative quantities $\tau_{\Pi}, \tau_{n}, \tau_{\pi}$. At this stage, it seems as though the three relaxation times $\tau_{\Pi}, \tau_{n}, \tau_{\pi}$ are all equal to $\tau_{R}$. This is because the collision term in the BE, Eq. (11) is written in RTA which does not entirely capture the microscopic interactions. This apparent equality vanishes if the first-order equation, Eq. (16) is compared with the relativistic Navier-Stokes equations for dissipative quantities $\left(\Pi=-\zeta \theta, n^{\mu}=\lambda T \nabla^{\mu} \alpha\right.$ and $\left.\pi^{\mu \nu}=2 \eta \sigma^{\mu \nu}\right)$. The dissipative relaxation times are then obtained in terms of first-order transport coefficients $\zeta, \lambda$ and $\eta$ which can be calculated independently taking into account the full microscopic behavior of the system [16, 17].

We remark that although the form of the evolution equations for dissipative quantities obtained here, Eqs. (24)-(26), are the same as those obtained in the previous calculations using both 14-moment approximation and second moment of BE [18], the coefficients obtained are different. In the following discussion, we refer to the results in Ref. [18] as the IS results although the power counting scheme differs from the one employed originally by IS.

For the special case of a system consisting of single species of massless Boltzmann gas, we find that

$$
\beta_{\pi}=\frac{4 P}{5}, \quad \tau_{\pi \pi}=\frac{10}{7}, \quad \delta_{\pi \pi}=\frac{4}{3} ;
$$

while these coefficients obtained via IS approach are [18]

$$
\beta_{\pi}^{\mathrm{IS}}=\frac{2 P}{3}, \quad \tau_{\pi \pi}^{\mathrm{IS}}=2, \quad \delta_{\pi \pi}^{\mathrm{IS}}=\frac{4}{3} .
$$

In this limit, although the coefficients of $\pi^{\mu \nu} \theta$ are same for both the cases $\left(\delta_{\pi \pi}=\delta_{\pi \pi}^{\text {IS }}\right)$, the coefficient of $\sigma^{\mu \nu}$ and $\pi_{\gamma}^{\langle\mu} \sigma^{\nu\rangle \gamma}$ are different $\left(\beta_{\pi} \neq \beta_{\pi}^{\mathrm{IS}}, \tau_{\pi \pi} \neq \tau_{\pi \pi}^{\mathrm{IS}}\right)$.

We note that CE expansion, as opposed to 14-moment approximation, can be done iteratively to arbitrarily 


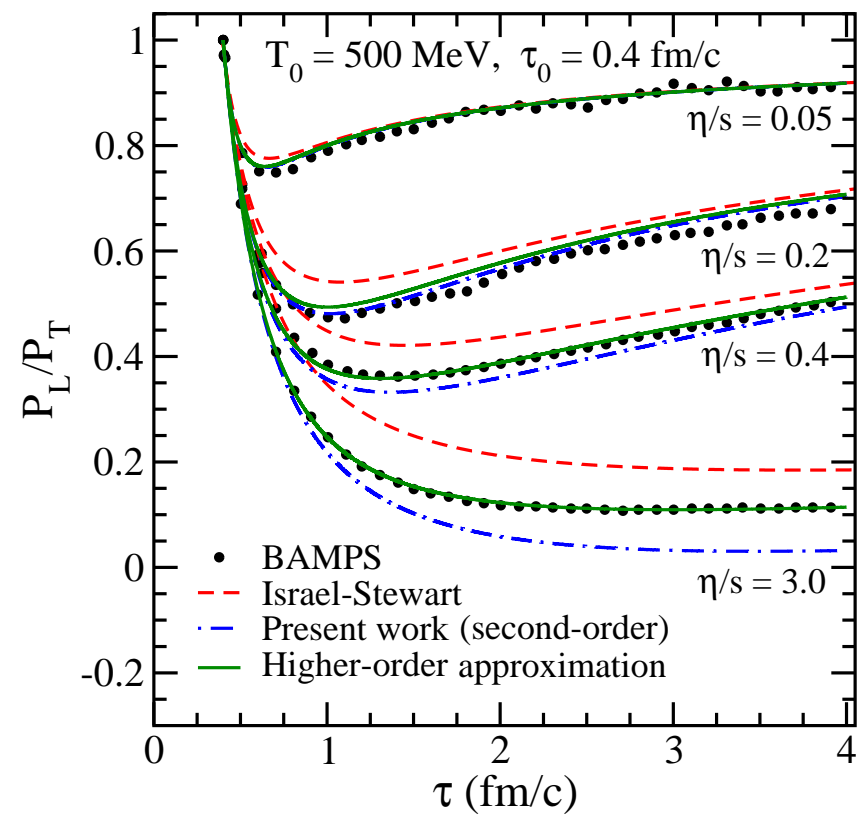

FIG. 1: (Color online) Time evolution of $P_{L} / P_{T}$ in BAMPS (dots), IS (dashed lines), present work (dashed-dotted line), and a heuristic higher-order approximation (solid line) for isotropic initial pressure configuration $\left(\pi_{0}=0\right)$.

higher orders. Hence using CE expansion, dissipative hydrodynamic equations of any order can in principle be derived. To obtain $n$ th-order evolution equations for dissipative quantities, $\delta f=\delta f^{(1)}+\delta f^{(2)}+\cdots+\delta f^{(n)}$ should be used in Eqs. (10)- (12). For instance, substitution of $\delta f=\delta f^{(1)}+\delta f^{(2)}+\delta f^{(3)}$ in Eqs. (10)-(12) will eventually lead to third-order evolution equations. Derivation of third-order hydrodynamics as outlined above is left for future work.

To demonstrate the numerical significance of the new coefficients derived here, we consider evolution in the boost invariant Bjorken case of a massless Boltzmann gas $(\epsilon=3 P)$ at vanishing net baryon number density [19]. In terms of the Milne co-ordinates $(\tau, x, y, \eta)$, where $\tau=\sqrt{t^{2}-z^{2}}$ and $\eta=\tanh ^{-1}(z / t)$, the initial four-velocity becomes $u^{\mu}=(1,0,0,0)$. For this scenario, $\Pi=n^{\mu}=0$, and the evolution equations for $\epsilon$, $\pi \equiv-\tau^{2} \pi^{\eta \eta}$ reduces to

$$
\begin{aligned}
& \frac{d \epsilon}{d \tau}=-\frac{1}{\tau}(\epsilon+P-\pi), \\
& \frac{d \pi}{d \tau}=-\frac{\pi}{\tau_{R}}+\beta_{\pi} \frac{4}{3 \tau}-\lambda \frac{\pi}{\tau} .
\end{aligned}
$$

The second-order transport coefficients simplify to

$$
\lambda \equiv \frac{1}{3} \tau_{\pi \pi}+\delta_{\pi \pi}=\frac{38}{21}, \quad \lambda^{\mathrm{IS}}=2 .
$$

Initial temperature $T_{0}=500 \mathrm{MeV}$ at proper time $\tau_{0}=0.4 \mathrm{fm} / \mathrm{c}$ are chosen to solve the coupled differential Eqs. (29) and (30). These values correspond to LHC initial conditions [20]. We assume isotropic initial pressure configuration i.e. $\pi_{0}=0$. Fig. 1 shows the proper time dependence of pressure anisotropy defined as $P_{L} / P_{T}=(P-\pi) /(P+\pi / 2)$. The dashed and dasheddotted lines represent the results from IS theory and our second-order results, respectively. The dots correspond to the results of a transport model, the Boltzmann Approach of MultiParton Scatterings (BAMPS), which is based on parton cascade simulations [9, 21]. The calculations in BAMPS are performed with variable values for the cross section such that the shear viscosity to entropy density ratio is a constant.

We note that the results from IS theory always overestimate the pressure anisotropy as compared to the transport results even for viscosities as small as $\eta / s=0.05$. It is evident from the figure that our results are in better agreement with BAMPS as compared to the results of IS. For very high viscosity, i.e., for $\eta / s=3.0$, although at early times we have a better agreement with BAMPS as compared to IS, at later times there is a large deviation. This disagreement may be attributed to the fact that the present hydrodynamic calculation is terminated at second-order in gradients. Inclusion of higher-order corrections may improve the agreement of dissipative hydrodynamic calculation results with those obtained using BAMPS as illustrated in the following.

In Ref. [9], while performing a third-order calculation it was demonstrated that within one-dimensional scaling expansion, the higher-order gradient terms can acquire the form $\left(\frac{\pi}{\epsilon}\right)^{n} \frac{\epsilon}{\tau}$, where, $n=r-1$ for $r$ th-order corrections. The other forms of higher-order corrections is reducible to this structure through lower-order evolution equations. Here we assume a similar heuristic expression for higher-order corrections

$$
\frac{d \pi}{d \tau}=-\frac{\pi}{\tau_{R}}+\beta_{\pi} \frac{4}{3 \tau}-\lambda \frac{\pi}{\tau}-\chi \frac{\pi^{2}}{\beta_{\pi} \tau}
$$

where the coefficient $\chi$ contains corrections to shear stress evolution due to higher-order gradients. This coefficient can be obtained by demanding that the above equation be valid for a free streaming of particles in the limit of infinite shear viscosity $(\eta \rightarrow \infty)$. In this limit, $\tau_{R} \rightarrow \infty$, and within one-dimensional scaling expansion the energy density evolves as $\dot{\epsilon}=-\epsilon / \tau$ which implies that $\dot{P}=-P / \tau$. For this case, using Eq. (29), we arrive at $\pi=\epsilon / 3=P$ which indicates disappearance of the longitudinal pressure. Substituting all these in Eq. (32), we obtain $\chi=36 / 175$.

Fig. 1, also shows $P_{L} / P_{T}$ evolution for the results obtained after including higher-order corrections (solid lines). We observe that the incorporation of higherorder corrections significantly improves the agreement with BAMPS. It is important to note that the BAMPS calculations are performed with the form of the collision term that captures the realistic microscopic interactions whereas the derivation of dissipative hydrodynamic equations in the present work uses RTA for the collision term. Within CE formalism, more sophisticated ways exist for solving the BE, for eg., by using variational methods [3] 
or by considering momentum dependent relaxation time [22, 23]. It is, in principle, possible to derive secondorder dissipative hydrodynamic evolution equations using momentum dependent relaxation time provided the dependence is specified explicitly. While this is left for future work, we observe that the near perfect agreement of the BAMPS results with those obtained using higherorder corrections clearly suggest that the momentum independent relaxation time for the BE used in the present derivation is sufficiently reliable for the range of $\eta / s$ considered here. However, the results obtained by using a momentum dependent relaxation time may show a better agreement with BAMPS data already at second-order.

RTA for the collision term assumes that the effect of the collisions is to restore the distribution function to its local equilibrium value exponentially. This is a very good approximation as long as the deviations from local equilibrium are small. As discussed above, we find that for the range of $\eta / s$ considered here, the deviation from equilibrium is not so large because the RTA is still valid. It is also important to note that large values of $\eta / s(>0.4)$ are not relevant to the physics of strongly coupled systems like Quark Gluon Plasma (QGP). The QGP formed at RHIC and LHC behaves as a near perfect fluid with a small estimated $\eta / s \approx 0.08-0.2$ [24, 25]. Using second-order evolution equations derived here, we get reasonably good agreement with BAMPS results for $\eta / s \leq 0.4$ (Fig. 11). This suggests that BE with RTA for the collision term can be successfully applied in understanding the hydrodynamic behaviour of QGP formed in relativistic heavy-ion collisions.

To summarize, we have presented a new derivation of relativistic second-order hydrodynamics from BE. We use Chapman-Enskog expansion for out of equilibrium distribution function instead of 14-moment approximation and derive evolution equations for dissipative quantities directly from their definitions rather than employing second moment of Boltzmann equation. In this new approach, we get rid of two powerful assumptions of IsraelStewart kind of derivation which is 14-moment approximation and choice of second moment of Boltzmann equation. Although the form of the evolution equation remains the same, the coefficients are found to be different. For small $\eta / s$, our second-order results show reasonably good agreement with the parton cascade BAMPS for the $P_{L} / P_{T}$ evolution. We find that heuristic inclusion of higher-order corrections in shear evolution equation significantly improves the agreement with transport calculation for large $\eta / s$ as well. This concurrence also suggests that relaxation time approximation for the collision term in Boltzmann equation is reasonably accurate when applied to heavy-ion collisions.

\section{Acknowledgments}

The author would like to thank Rajeev S. Bhalerao and Subrata Pal for fruitful discussions, critical reading of the manuscript and helpful comments.
[1] C. Eckart, Phys. Rev. 58, 267 (1940).

[2] L.D. Landau and E.M. Lifshitz, Fluid Mechanics (Butterworth-Heinemann, Oxford, 1987).

[3] S. Chapman and T. G. Cowling, The Mathematical Theory of Non-Uniform Gases, (Cambridge University Press, Cambridge, 1970), 3rd ed.

[4] W. Israel and J. M. Stewart, Annals Phys. 118, 341 (1979).

[5] P. Huovinen and D. Molnar, Phys. Rev. C 79, 014906 (2009).

[6] G. S. Denicol, T. Koide and D. H. Rischke, Phys. Rev. Lett. 105, 162501 (2010).

[7] A. Jaiswal, R. S. Bhalerao and S. Pal, Phys. Rev. C 87, 021901 (2013)

[8] A. Jaiswal, R. S. Bhalerao and S. Pal, Phys. Lett. B 720, 347 (2013)

[9] A. El, Z. Xu and C. Greiner, Phys. Rev. C 81, 041901 (2010).

[10] G. S. Denicol, H. Niemi, E. Molnar and D. H. Rischke, Phys. Rev. D 85, 114047 (2012).

[11] J. L. Anderson and H. R. Witting Physica 74, 466 (1974).

[12] P. Romatschke, Phys. Rev. D 85, 065012 (2012).

[13] H. Grad, Comm. Pure Appl. Math. 2, 331 (1949).
[14] S.R. de Groot, W.A. van Leeuwen, and Ch.G. van Weert, Relativistic Kinetic Theory - Principles and Applications (North-Holland, Amsterdam, 1980).

[15] See Supplemental Material in the source file of arXiv:1302.6311 for details of the derivation.

[16] P. Kovtun, D. T. Son and A. O. Starinets, Phys. Rev. Lett. 94, 111601 (2005).

[17] H. B. Meyer, Phys. Rev. Lett. 100, 162001 (2008).

[18] B. Betz, D. Henkel and D. H. Rischke, Prog. Part. Nucl. Phys. 62, 556 (2009), J. Phys. G 36, 064029 (2009).

[19] J. D. Bjorken, Phys. Rev. D 27, 140 (1983).

[20] A. El, Z. Xu and C. Greiner, Nucl. Phys. A 806, 287 (2008)

[21] Z. Xu and C. Greiner, Phys. Rev. C 71, 064901 (2005).

[22] M. Prakash, M. Prakash, R. Venugopalan and G. Welke, Phys. Rept. 227, 321 (1993).

[23] D. A. Teaney, arXiv:0905.2433 [nucl-th].

[24] P. Romatschke and U. Romatschke, Phys. Rev. Lett. 99 (2007) 172301.

[25] H. Song, S. A. Bass, U. Heinz, T. Hirano and C. Shen, Phys. Rev. Lett. 106 (2011) 192301. 